\title{
Composição da comunidade liquênica em floresta ribeirinha na APA do Ibirapuitã, RS, Brasil
}

\author{
Márcia Isabel Käffer ${ }^{1,3}$, Suzana Maria de Azevedo Martins ${ }^{1}$, Renata Villar Dantas ${ }^{1,2} \mathrm{e}$ \\ Felipe Coutinho Maciel ${ }^{1,2}$
}

Recebido: 22.09.2014; aceito: 11.02.2015

\begin{abstract}
Lichen community composition in riparian forest in the APA of Ibirapuitã, Rio Grande do Sul State, Brazil). The lichens are part of an extremely diverse group and are important components in forested areas. The aim of this study is to analyze the lichen species composition and investigate the phytosociological patterns of taxa occurring in riparian forest, in the APA of Ibirapuitã, Rio Grande do Sul State, Brazil. The mapping of lichen species was performed using the acetate method, in 60 phorophytes distributed in 12 riparian forest areas. Two hundred fifty-four lichenized fungi taxa were recorded, of which 199 were registered in the quantitative study, with two new species to science, three new citations for Brazil and 14 new records for the state of Rio Grande do Sul. The species Heterodermia obscurata (Nyl.) Trev. was the most important in the community, showing the highest value of importance, frequency, and coverage. The environmental quality of some riparian forest areas, associated with the availability of appropriate phorophyte and microclimate conditions of forest areas are factors that contributed to the results. The preservation of riparian forests in the analyzed areas is of vital importance to ensure the biodiversity of species, but also for the protection of watercourses of Ibirapuitã River and its tributaries.
\end{abstract}

Keywords: corticicolous lichens, lichenized fungi, Pampa biome, phytosociology

RESUMO - (Composição da comunidade liquênica em floresta ribeirinha na APA do Ibirapuitã, RS, Brasil). Os liquens fazem parte de um grupo extremamente diverso e são componentes importantes em áreas florestais. O objetivo deste trabalho foi analisar a composição da comunidade liquênica e verificar os padrões fitossociológicos de táxons ocorrentes em áreas florestais ribeirinhas, na APA do Ibirapuitã, RS, Brasil. O mapeamento dos liquens foi realizado através do método do acetato, em 60 forófitos (palavra masculina que designa árvores que servem de suporte para epífitas) distribuídos em 12 áreas florestais. Foram registrados 254 táxons liquênicos, destes 199 registrados no estudo quantitativo com duas novas espécies para a ciência, três novas citações para o Brasil e 14 novos registros para o Estado do Rio Grande do Sul. A espécie Heterodermia obscurata (Nyl.) Trev. foi a mais importante na comunidade, apresentando maior valor de importância, frequência e cobertura. A boa qualidade ambiental de algumas áreas florestais ribeirinhas, associada à disponibilidade de forófitos adequados e as condições microclimáticas das áreas florestais são fatores que contribuíram para os resultados encontrados. A preservação das florestas ribeirinhas nas áreas analisadas é de vital importância para assegurar a diversidade das espécies como também para a proteção dos cursos d' água do rio Ibirapuitã e seus afluentes.

Palavras-chave: bioma Pampa, fitossociologia, fungos liquenizados, liquens corticícolas

\section{Introdução}

As modificações na paisagem das florestas e as práticas de manejo influenciam fortemente a biodiversidade, tanto em termos de composição e riqueza de espécies como no tamanho das populações. Os liquens, especialmente as espécies ocorrentes em áreas florestais antigas são os mais afetados entre os grupos de organismos, reagindo inclusive por pequenas alterações na estrutura do seu habitat (Richardson \& Cameron 2004), sendo utilizados como indicadores de estágios florestais.

Estes organismos são mais desenvolvidos em florestas antigas do que em florestas jovens e permitem demonstrar se o ecossistema florestal permaneceu inalterado ao longo do tempo (McCune 1993, Esseen

1. Fundação Zoobotânica do Rio Grande do Sul, Museu de Ciências Naturais, Caixa postal 1188, 90690-000 Porto Alegre, RS, Brasil

2. Pontifícia Universidade Católica do Rio Grande do Sul, Curso de Ciências Biológicas, 90610-000 Porto Alegre, RS, Brasil

3. Autor de correspondência: m.kaffer@terra.com.br 
et al. 1996, Tuvi et al. 2011, Nascimbene et al. 2013). Mesmo que algumas comunidades sejam capazes de sobreviver a este tipo de impacto, muitas outras podem sofrer extinção local de espécies, as quais são restritas aos ecossistemas com baixos regimes de perturbações (Werth 2001). Em algumas comunidades pode ocorrer perda de diversidade e/ou se encontrar dominadas por espécies mais resistentes a ambientes perturbados ou poluídos (Brunialti \& Giordani 2003, Jüriado et al. 2003).

Os campos temperados do sul da América do Sul (Bioma Pampa) originalmente abrigavam uma área de mais de um milhão de $\mathrm{km}^{2}$; no entanto, um pequeno percentual permanece em estado natural e o pouco do que resta está ameaçado pela intensificação agrícola (WBW 2012). Esse bioma abriga alta biodiversidade, com cerca de duas mil espécies vegetais (Boldrini 2009); entretanto, o conhecimento de outros grupos biológicos ainda é escasso.

Os liquens fazem parte de um grupo extremamente diverso e exercem diferentes funções nos ecossistemas. Servem de habitat para alguns animais e contribuem para reciclagem de nutrientes, com a fixação de nitrogênio pelas cianobactérias associadas (Brunialti \& Giordani 2003, Gunnarsson et al. 2004).

No Brasil, são poucos os estudos que abordam os efeitos ambientais em áreas florestais sobre a comunidade liquênica (Käffer \& Martins-Mazzitelli 2005, Cáceres et al. 2008, Fleig \& Grüninger 2008, Käffer et al. 2009, 2010, Martins \& Marcelli 2011, Koch et al. 2012).

Para a área do Bioma Pampa, Käffer \& Martins (2014) analisaram a qualidade ambiental de algumas áreas florestais empregando os liquens como indicadores ambientais, uma vez que estes são considerados organismos pioneiros na colonização de ambientes (Valencia \& Ceballos 2002) e sensíveis às variações ambientais (Giordani et al. 2012). Desta forma, este trabalho tem por objetivos: i) analisar a composição da comunidade liquênica corticícola de áreas florestais ribeirinhas na região sul da APA do Ibirapuitã; e ii) verificar a frequência, cobertura e valor de importância dos táxons identificados.

\section{Material e métodos}

Área de estudo - A Área de Proteção Ambiental (APA) do Ibirapuitã é uma Unidade de Conservação Federal da Categoria "Uso Sustentável". Possui uma extensão de, aproximadamente, 317.000 hectares representativos do Bioma Pampa, sendo a única UC
Federal deste Bioma. Seu território está distribuído em quatro municípios do Estado do Rio Grande do Sul, Brasil: Alegrete (15\%), Quaraí (12\%), Rosário do Sul (16\%) e Santana do Livramento (57\%). A vegetação predominante é o campo e, ao longo dos rios, há a presença de floresta de galeria; nas demais áreas, remanescentes de floresta de encosta e capões de mata (Backes 2012, Andrade 2013).

O presente trabalho foi realizado no período de junho de 2011 a março de 2013. Foram analisadas 12 áreas amostrais, situadas na região sul da APA do Ibirapuitã, em Santana do Livramento: Fazenda Lolita (LO1-LO6), Estância São Maurício (MA1-MA3) e Fazenda Rincão Bonito (RB1-RB3) (figura 1).

Amostragem e identificação - Para o estudo da comunidade liquênica foram empregadas análise quali-quantitativa. No estudo qualitativo, os liquens foram observados e/ou coletados em substratos (árvores, rochas, solo) através do método de caminhadas (Filgueiras et al. 1994), por trilhas e acessos localizados junto e/ou próximo às matas ribeirinhas, nas mesmas áreas do estudo quantitativo.

$\mathrm{Na}$ amostragem quantitativa, em cada área amostrada foram selecionados cinco forófitos, preferencialmente de troncos eretos, que não apresentassem ramificações abaixo de $150 \mathrm{~cm}$ de altura e com diâmetro altura do peito (DAP) acima de $7,3 \mathrm{~cm}$, totalizando 60 forófitos.

Para o mapeamento da micota liquenizada foi utilizado o método das folhas de acetato. Este consiste em dispor cinco folhas de acetato de $20 \times 20 \mathrm{~cm}$ ao longo do tronco dos forófitos, a partir de $50 \mathrm{~cm}$ acima do solo até $150 \mathrm{~cm}$, amostrando o tronco em cinco níveis de altura, nas faces Norte e Sul dos mesmos.

Os liquens não identificados no local foram coletados para posterior confirmação em laboratório. Para a identificação das espécies foi utilizado o procedimento padrão em liquenologia. Foram realizadas análises morfológicas através da identificação dos caracteres macro e microscópicos dos espécimes, com o uso de estereomicroscópio e de microscópio óptico. As análises químicas foram realizadas através dos testes de spot baseados na coloração, utilizando-se reagentes químicos, e de luz UV (ondas longas) no córtex e medula das amostras para constatação de substâncias (ácidos liquênicos).

Também foi utilizada bibliografia especializada para cada grupo taxonômico, consulta com material constante no Herbário Prof. Dr. Alarich Schultz (HAS) do Museu de Ciências Naturais da Fundação Zoobotânica do Rio Grande do Sul, Brasil. A 
confirmação de algumas espécies foi realizada por especialistas, para alguns grupos. O material coletado encontra-se herborizado e catalogado no herbário HAS (89232 a 89436).

Análise dos dados fitossociológicos - A riqueza de espécies foi considerada como o número total de liquens ocorrentes nos cinco forófitos. A estimativa da frequência baseou-se na presença $\mathrm{x}$ ausência das espécies nos troncos dos forófitos, de todas as áreas de amostragens. A estimativa da cobertura para cada espécie foi realizada através da soma total da cobertura de todos os talos presentes, em cada uma das cinco folhas de acetato, de todos os forófitos analisados. A abundância por espécie foi considerada como a soma da contagem individual de cada espécime que ocorreu nas folhas de acetato, nos cinco níveis de altura do tronco dos forófitos amostrados. O valor de importância (VI) de cada espécie foi calculado em relação à comunidade como um todo, somando-se os dados de frequência e cobertura relativa.

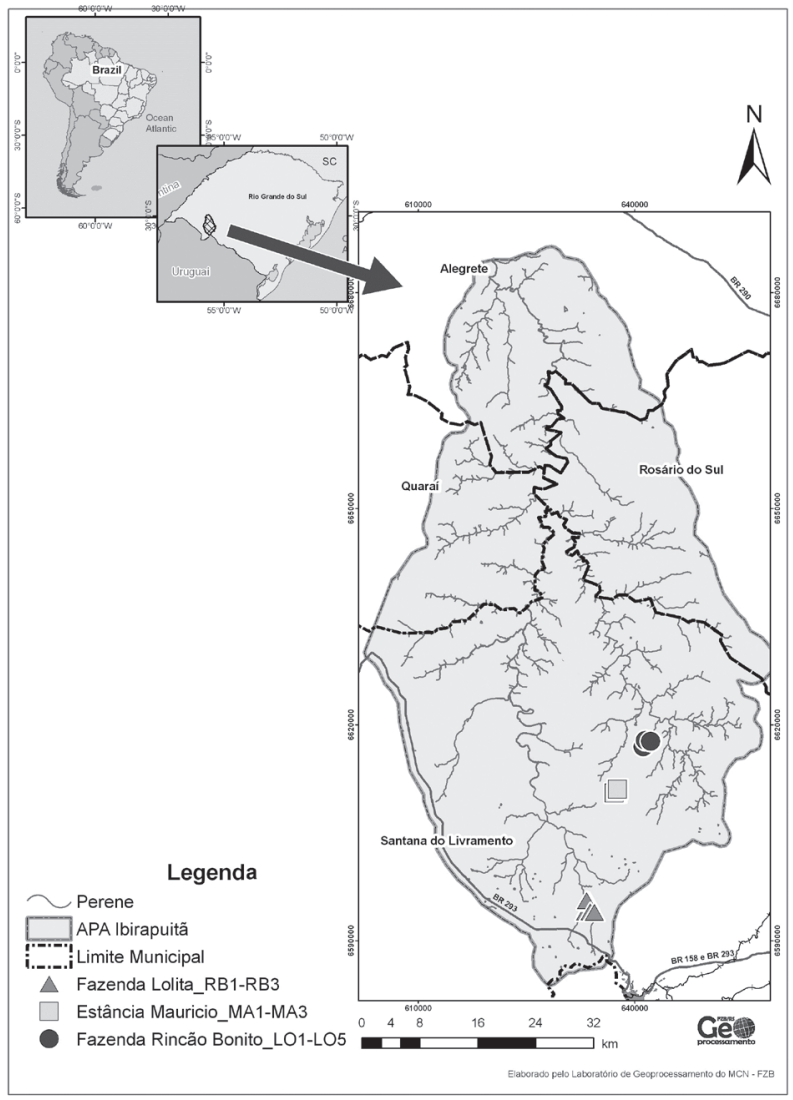

Figura 1. Mapa de localização das áreas de estudo da comunidade liquênica, na região sul da APA do Ibirapuitã, Santana do Livramento, RS, Brasil.

Figure 1. Location map of the areas of study of lichenized community in the southern region of the APA do Ibirapuitã, Santana do Livramento, Rio Grande do Sul State, Brazil.

\section{Resultados}

Florística - No estudo qualitativo foram identificadas 254 espécies liquênicas distribuídas em 71 gêneros e 27 famílias (tabela 1). Duas espécies são novas para ciência, as quais estão sendo analisadas por especialistas do grupo: Chapsa sp. e Pseudocyphellaria sp. Três espécies são citadas aqui pela primeira vez para o Brasil: Coenogonium byssothallinum Aptroot \& Lücking, Hyperphyscia viridissima (Müll. Arg.) Scutari e Phyllopsora cf. santensis (Tuck.) Swinsc. $\&$ Krog.

Para o Estado do Rio Grande do Sul, 14 são novos registros: Coenogonium pyrophthalmum (Mont.) Lücking, Aptroot \& Sipman, Hafelia cf. curatelliae (Malme) Marbach, Heterodermia africana (Kurok.) M.P. Marcelli \& M.F.N. Martins, Heterodermia tremulans (Müll.Arg.) W.L. Culb, Hypotrachyna sublaevigata (Nyl.) Hale, Leptogium corticola (Taylor) Tuck., Leptogium diaphanum (Mont.) Nyl., Leptogium sp. 1, Leptogium sp. 3, Physcia sinuosa Moberg., Punctelia crispa Marcelli, Jungbluth \& Elix., Punctelia fimbriata Marcelli \& Canêz, Punctelia sp. 3 e Punctelia sp. 4.

Do total das espécies, $92,9 \%$ são colonizadas por clorofíceas e 7,1\% por cianofíceas. Quanto ao hábitat, o maior predomínio foi de espécies corticícolas (95\%) e as demais ocorrentes em habitat saxícola, muscícola e terrícola.

Em relação ao hábito foi registrado maior percentual de espécies foliosas $(50 \%)$, seguido do grupo morfológico crostoso $(35,4 \%)$, fruticoso $(9,4 \%)$, filamentoso (1,6\%), esquamuloso $(2,4 \%)$ e dimórfico $(1,2 \%)$. As famílias mais representativas, em relação à riqueza de espécies, foram Parmeliaceae (29,5\%), Physciaceae (16,5\%) e Graphidaceae (8,3\%). Os gêneros com maior número de espécies foram Parmotrema (24), Punctelia (18), Heterodermia (17) e Usnea (16).

Estudo quantitativo - Foram registradas 199 espécies liquênicas distribuídas em 52 gêneros com 22 famílias. Parmeliaceae, Physciaceae, Graphidaceae e Ramalinaceae foram as famílias com maior número de espécies (61,33, 19 e 17), sendo os gêneros com maior número de espécies: Parmotrema (24), Punctelia (16), Usnea (13) e Physcia (11) (tabela 2).

A análise da composição demonstrou que a soma dos VIs dos cinco táxons com maior valor de importância representou 77,6\% do VI total $\left(\sum=200\right)$. A espécie Heterodermia obscurata apresentou o maior valor de importância da comunidade (VI = 31,28), o 
Tabela 1. Famílias e espécies liquênicas registradas nas áreas florestais ribeirinhas, na região sul da APA do Ibirapuitã, RS, Brasil. Espécies novas para a ciência $(*)$, novos registros para o Brasil $(* *)$ e novas citações para o RS $(* * *)$.

Table 1. Families and species lichenized recorded in riparian forest areas in the southern region of the APA do Ibirapuitã, Rio Grande do Sul State, Brazil. Species new to science $(*)$, new record for Brazil (**) and new citations for the RS (***).

\section{Famílias/Espécies}

Arthoniaceae

\section{Arthonia sp. 1}

Arthonia sp. 2

Herpothallon rubrocinctum (Ehrenb.) Aptroot \& Lücking

Brigantiaceae

Brigantiaea leucoxantha (Spreng.) R. Sant. \& Haf.

Coccocarpiaceae

Cococcarpia erythroxyli (Spreng.) Sw. \& Krog

Cococcarpia pellita (Ach.) Müll.Arg.

Cococcarpia stellata Tuck

Coenogoniaceae

Coenogonium byssothalinum Aptroot \& Lücking**

Coenogonium interplexum Nyl.

Coenogonium linkii Ehrenb.

Coenogonium nepalense (G.Thor \&Vězda) Lücking, Aptroot \& Sipman

Coenogonium pyrophthalmum (Mont.) Lücking, Aptroot \& Sipman***

Coenogonium subdilutum (Mont.) Lücking, Aptroot \& Sipman

Chrysothrichaceae

Chrysothrix sp.

Cladoniaceae

Cladonia ceratophylla (Sw.) Spreng.

Cladonia chlorophaea (Flörke ex Sommerf.) Spreng.

Cladonia cf. sphacelata Vain.

\section{Collemataceae}

Collema fasciculare (L.) Wigg.

Leptogium austroamericanum (Malme) C. W. Dodge

Leptogium azureum (Sw. ex Ach.) Mont.

Leptogium cochleatum (Dickson) P.M. Jorg. \& P. James

Leptogium corticola (Taylor) Tuck.***

Leptogium cyanescens (Robenhorst) Körber

Leptogium denticulatum Nyl.

Leptogium diaphanum (Mont.) Nyl.***

Leptogium involutum Kitaura, Käffer \& S.M.Martins

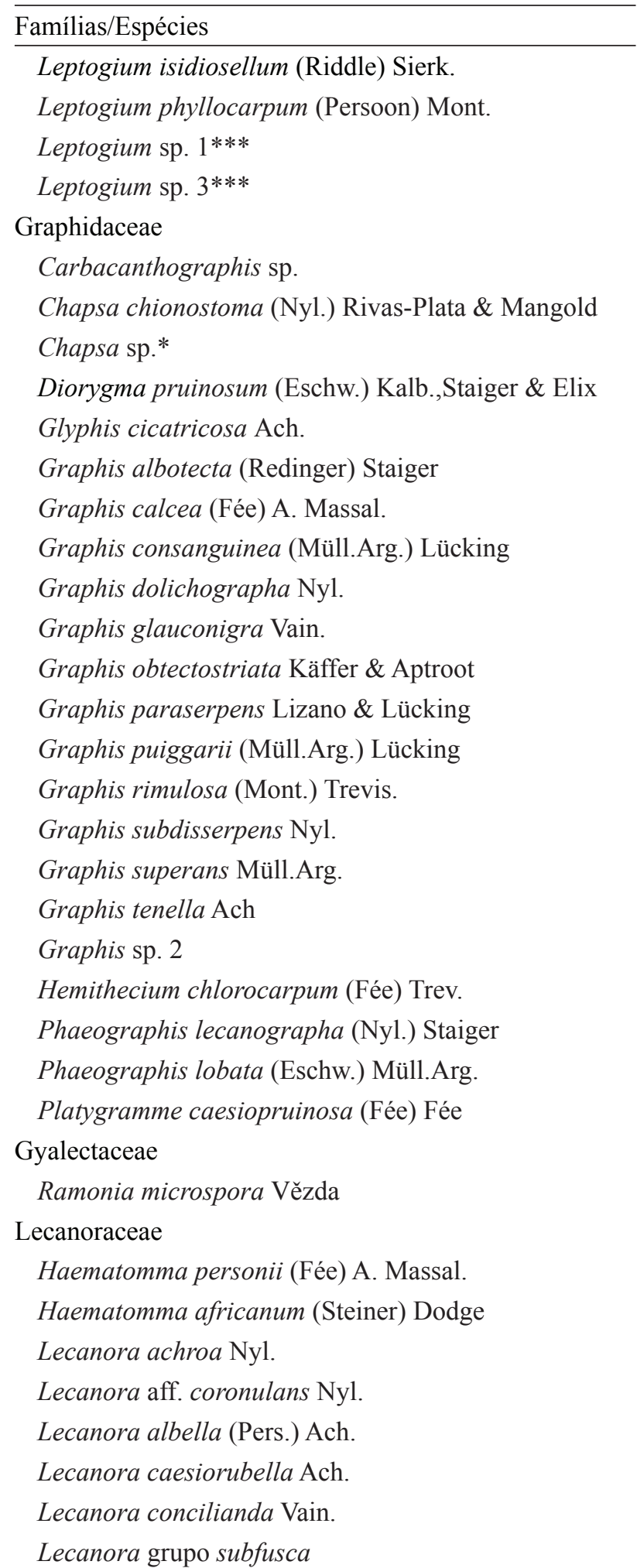

continua 
Tabela 1 (continuacão)

Famílias/Espécies

Lecanora hypocrocina $\mathrm{Nyl}$.

Lecanora thysanophora R.C. Harris

Protoparmelia multifera (Nyl.) Kantvilas, Papong \& Lumbsch

Ramboldia haematites (Fée) Kalb

Tephromela americana (Fée) Kalb

Lobariaceae

Ricasolia cuprea Müll.Arg.

Ricasolia discolor (Bory) Nyl.

Ricasolia erosa (Eschw.) Nyl.

Ricasolia intermedia Nyl.

Ricasolia patinifera (Taylor) Müll.Arg.

Monoblastiaceae

Anisomeridium albisedum (Nyl.) R.C.Harris

Anisomeridium leptospermum (Zahlbr.) R.C.Harris

Parmeliaceae

Bulbothrix subcoronata (Müll.Arg.) Hale

Crespoa carneopruinata (Zahlbr.) Elix \& Hale

Canoparmelia caroliniana (Nyl.) Elix \& Hale

Canoparmelia roseoreagens Marcelli \& Canêz

Canoparmelia texana (Tuck.) Elix \& Hale

Flavoparmelia exornata (Zahlbr.) Hale

Flavoparmelia rutidota (Hook \& Taylor) Hale

Hypotrachyna degelii (Hale) Hale

Hypotrachyna intercalanda (Vain.) Hale

Hypotrachyna livida (Taylor) Hale

Hypotrachyna pluriformis (Nyl.) Hale

Hypotrachyna sublaevigata (Nyl.) Hale ***

Parmelinopsis horrescens (Taylor) Elix \& Hale

Parmelinopsis minarum (Vain.) Elix \& Hale

Parmelinopsis sp.

Parmotrema catarinae Hale

Parmotrema cetratum (Ach.) Hale

Parmotrema clavuliferum (Räsänen) Streimann

Parmotrema commensuratum (Hale) Hale

Parmotrema consors (Nyl.) Krog \& Swinsc.

Parmotrema ecilatum (Nyl.) Hale

Parmotrema eurysacum (Hue) Hale

Parmotrema haitiense (Hale) Hale
Tabela 1 (continuação)

Famílias/Espécies

Parmotrema internexum (Nyl.) Hale ex De Priest \& B.W. Hale

Parmotrema melanothrix (Mont.) Hale

Parmotrema mellissii (Dodge) Hale

Parmotrema muelleri (Vain.) Blanco, Crespo, Divakar, Elix \& Lumbsch

Parmotrema lindmanii (Lynge) Kurok.

Parmotrema pilosum (Stizenb.) Krog \& Swinsc.

Parmotrema praesorediosum (Nyl.) Hale

Parmotrema recipiendum (Nyl.) Hale

Parmotrema reticulatum (Taylor) M. Choisy

Parmotrema rigidum (Lynge) Hale

Parmotrema ruptum (Lynge) Hale

Parmotrema sancti-angeli (Lynge) Hale

Parmotrema simulans (Hale) Hale

Parmotrema subcaperatum (Kremp.) Hale

Parmotrema subrugatum (Kremp.) Hale

Parmotrema tinctorum (Nyl.) Hale

Punctelia punctilla (Hale) Krog

Punctelia bolliana (Müll.Arg.) Krog

Punctelia colombiana Sérusiaux

Punctelia constantimontium Sérus.

Punctelia crispa Marcelli, Jungbluth \& Elix***

Punctelia fimbriata Marcelli \& Canêz***

Punctelia graminicola (B. de Lesd.) Egan

Punctelia hypoleucites Nyl.

Punctelia microsticta (Müll.Arg.) Krog

Punctelia osorioi Canêz \& Marcelli

Punctelia riograndensis (Lynge) Krog

Punctelia subpraesignis (Nyl.) Krog

Punctelia sp. 1

Punctelia sp. 2

Punctelia sp. $3^{* * *}$

Punctelia sp. $4^{* * *}$

Punctelia sp. 5

Punctelia sp. 6

Usnea angulata Ach.

Usnea florida (L.) Wigg. 
Tabela 1 (continuação)

Famílias/Espécies

Usnea rubicunda Stirton

Usnea cf. rubicunda Stirton

Usnea sp. 1

Usnea sp. 2

Usnea sp. 3

Usnea sp. 4

Usnea sp. 5

Usnea sp. 6

Usnea sp. 7

Usnea sp. 8

Usnea sp. 9

Usnea sp.10

Usnea sp.11

Usnea sp. 12

Xanthoparmelia elixii Filson

Peltigeraceae

Peltigera autroamericana Zahlbr.

Pertusariaceae

Ochrolechia africana Vain.

Pertusaria carneola (Eschw.) Müll.Arg.

Pertusaria flavens Nyl.

Pertusaria velata (Turner) Nyl.

Pertusaria sp. 1

Pertusaria sp. 2

Pertusaria sp. 3

Pertusaria sp. 4

Pertusaria sp. 5

Pertusaria sp. 6

Physciaceae

Cratiria obscurior (Stirton) Marbach

Dirinaria confluens (Fr.) Awasthi

Dirinaria picta (Sw.) Clem. \& Shear

Hafellia bahiana (Malme) Sheard

Hafelia cf. curatelliae (Malme) Marbach***

Hafelia demutans (Stirton) Pubwald

Heterodermia albicans (Pers.) Sw. \& Krog

Heterodermia africana (Kurok.) M.P. Marcelli \& M.F.N. Martins***

Heterodermia casarettiana (Massal.) Trev.
Tabela 1 (continuação)

Famílias/Espécies

Heterodermia comosa (Eschw.) Follm. \& Redón

Heterodermia diademata (Taylor) Awasthi

Heterodermia flabellata (Fée) Awasthi

Heterodermia flavosquamosa Aptroot \& Sipman

Heterodermia hypochraea (Vain.) Swinsc. \& Krog

Heterodermia japonica (Sato) Swinsc. \& Krog

Heterodermia leucomela (L.) Poelt

Heterodermia lutescens (Kurok.) Follm.

Heterodermia magellanica (Zahlbr.) Swinsc. \& Krog

Heterodermia obscurata (Nyl.) Trevis.

Heterodermia pseudospeciosa (Kurok.) W.L. Culb

Heterodermia speciosa (Wulf.) Trev.

Heterodermia tremulans (Müll.Arg.) W.L. Culb. ***

Heterodermia vulgaris (Vain.) Follmann \& Redón

Hyperphyscia adglutinata (Flörke) Maryhofer \& Poelt

Hyperphyscia syncolla (Tuck. ex Nyl.) Kalb

Hyperphyscia viridissima (Müll.Arg.) Scutari **

Phaeophyscia hispidula (Ach.) Moberg.

Physcia aipolia (Humb.) Fürnr.

Physcia alba (Fée) Müll.Arg.

Physcia atrostriata Moberg

Physcia crispa Nyl.

Physcia erumpens Moberg

Physcia krogiae Moberg

Physcia poncinsii Hue

Physcia sinuosa Moberg***

Physcia sorediosa (Vain.) Lynge

Physcia tribacoides Nyl.

Physcia undulata Moberg

Physciaceae

Pyxine cocoës (Sw.) Nyl.

Pyxine daedalea Krog \& Santesson

Pyxine subcinerea Stirton

Rinodina conradii Körb.

Pilocarpaceae

Calopadia pruinosa Lücking \& Chaves

Malmidea vinosa (Eschw.) Kalb, Rivas Plata \& Lumbsch

Malmidea fuscella (Müll.Arg.) Kalb \& Lücking

Malmidea hypomela (Nyl.) Cáceres \& Lücking 
Tabela 1 (continuação)

Famílias/Espécies

Porinaceae

Porina africana Müll.Arg.

Porina cf. simulans Müll.Arg.

Trichothelium horridulum (Müll.Arg.) R. Sant.

Trichothelium angustiporum Cáceres \& Lücking

Pyrenulaceae

Anthracothecium prasinum (Eschw.) R.C. Harris

Pyrenula mucosa (Vain.) R.C. Harris

Pyrenula pyrenuloides (Mont.) R.C.Harris

Pyrenula dissimulans (Müll.Arg.) R.C.Harris

Ramalinaceae

Bacidia fluminensis (Malme) Cáceres \& Lücking

Bacidia cf. heterochroa (Müll.Arg.) Zahlbr.

Bacidia russeola (Kremp.) Zahlbr.

Bacidia subtestacea Malme

Bacidia chapadensis Malme

Bapalmuia lafayettiana (Vain.) Kalb \& Lücking

Phlyctella brasiliensis (Nyl.) Nyl.

Phyllopsora breviuscula (Nyl.) Müll.Arg.

Phyllopsora buettneri (Müll.Arg.) Zahlbr.

Phyllopsora cf. santensis (Tuck.) Swinsc. \& Krog **

Phyllopsora furfuraceae (Pers.) Zahlbr.

Ramalina celastri (Spreng.) Krog \& Swinsc.

Ramalina peruviana Ach.

Ramalina puiggarii Müll.Arg.

Ramalina sprengelli Krog \& Swinsc.

Ramalina usnea (L.) Howe

Roccellaceae

Bactrospora jenikii (Vězda) Egea \& Torrente

Opegrapha sp. 1

maior número de indivíduos $(\mathrm{NI}=390)$ e altos valores de cobertura $(\mathrm{CA}=1069,2)$. Punctelia $\mathrm{sp} .5$ foi a segunda espécie mais importante na comunidade $(\mathrm{VI}=13,44)$, porém, apresentou menor número de indivíduos em relação as outras espécies $(\mathrm{NI}=135)$. Ricasolia erosa foi a terceira espécie com maior valor de importância $(\mathrm{VI}=11,09)$, porém foi registrado um menor número de indivíduos $(\mathrm{NI}=105)$. As espécies Parmotrema cetratum e Crespoa carneopruinata obtiveram o quarto e quinto maiores lugares na comunidade, apresentando valores de importância de 11,05 e 10,77, respectivamente. No entanto, estas tiveram uma variação no número de
Tabela 1 (continuação)

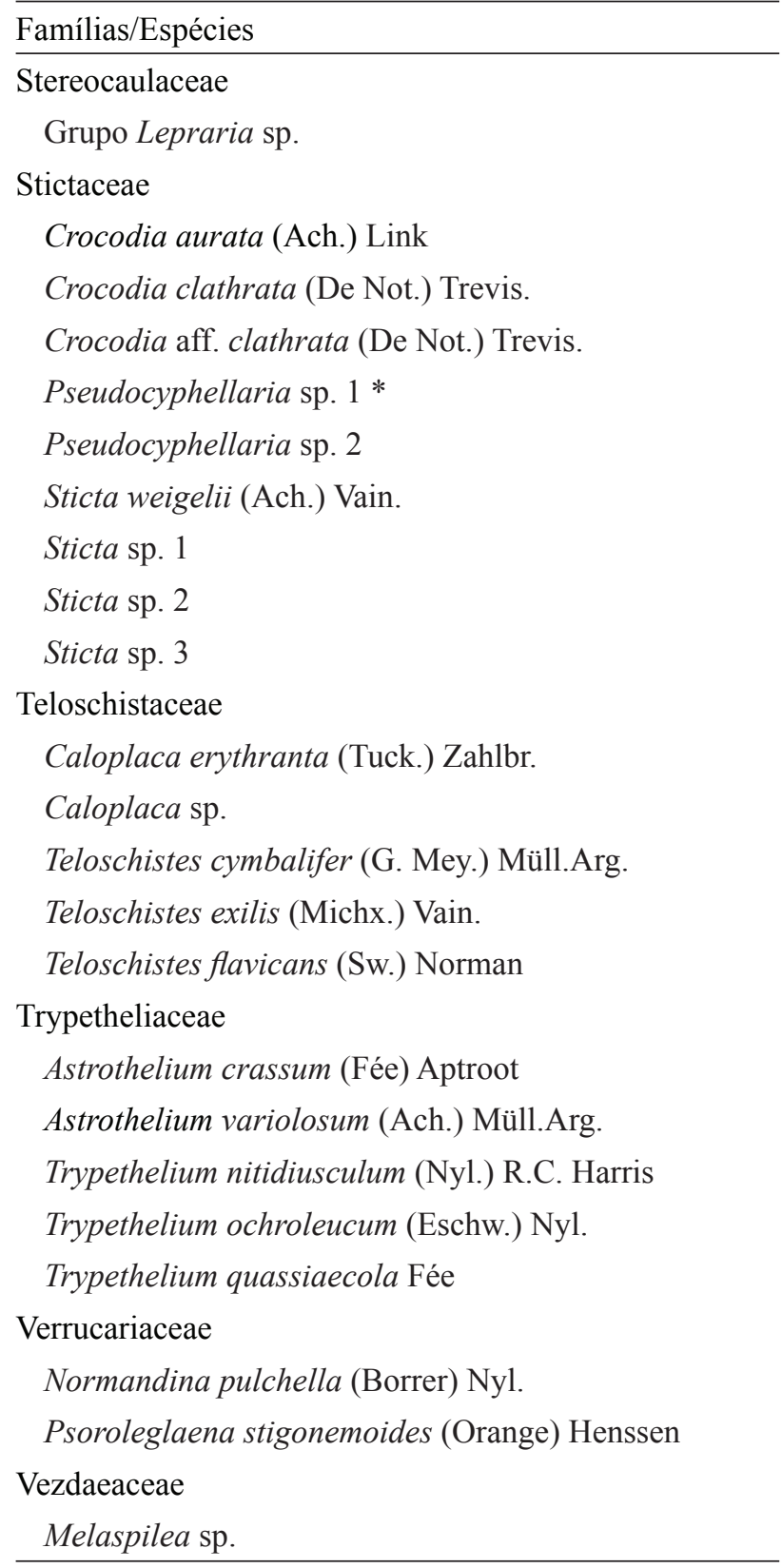

indivíduos em relação à $R$. erosa, NI = 161 e NI = 141 respectivamente (tabela 2).

Do total de espécies registradas no estudo quantitativo, o grupo morfológico crostoso representou $28,07 \%$ do VI total $\left(\sum=200\right)$. Deste grupo, Herpothallon rubrocinctum obteve o maior número de indivíduos $(\mathrm{NI}=48)$ e frequencia relativa $(\mathrm{FR}=1,71)$, seguida de Diorygma pruinosum $(\mathrm{NI}=46, \mathrm{FR}=1,64)$. Dos representantes da família Graphidaceae, se destaca a espécie Graphis obtectostriata que apresentou o segundo maior valor de importância $(\mathrm{VI}=1,68)$ e número de indivíduos $(\mathrm{NI}=22)$. 
Tabela 2. Parâmetros fitossociológicos da comunidade liquênica nas áreas florestais ribeirinhas,- na região sul da APA do Ibirapuitã, RS, Brasil. NI: número de indivíduos amostrados; CA: cobertura absoluta; CR: cobertura relativa; FA: frequência absoluta; FR: frequência relativa;VI: valor de importância.

Table 2. Phytosociological parameters of the lichenized community in the riparian forest areas in the southern region of APA do Ibirapuitã, Rio Grande do Sul State, Brazil. NI: number of individuals sampled; CA: absolute cover; CR: relative cover; FA: absolute frequency; FR: relative frequency; VI: value of importance

\begin{tabular}{|c|c|c|c|c|c|c|}
\hline Espécies & NI & $\mathrm{CA}$ & $\mathrm{CR}$ & FA & FR & VI \\
\hline Heterodermia obscurata & 390 & 1069,2 & 17,41 & 6,50 & 13,87 & 31,28 \\
\hline Punctelia sp. 5 & 135 & 530,3 & 8,63 & 2,25 & 4,80 & 13,44 \\
\hline Ricasolia erosa & 105 & 451,5 & 7,35 & 1,75 & 3,74 & 11,09 \\
\hline Parmotrema cetratum & 161 & 327,0 & 5,32 & 2,68 & 5,73 & 11,05 \\
\hline Crespoa carneopruinata & 141 & 353,2 & 5,75 & 2,35 & 5,02 & 10,77 \\
\hline Parmotrema reticulatum & 102 & 261,9 & 4,26 & 1,70 & 3,63 & 7,89 \\
\hline Ramalina peruviana & 68 & 140,7 & 2,29 & 1,13 & 2,42 & 4,71 \\
\hline Physcia atrostriata & 82 & 106,4 & 1,73 & 1,37 & 2,92 & 4,65 \\
\hline Crocodia aurata & 69 & 74,4 & 1,21 & 1,15 & 2,45 & 3,67 \\
\hline Punctelia sp. 1 & 34 & 149,5 & 2,43 & 0,57 & 1,21 & 3,64 \\
\hline Punctelia sp. 2 & 40 & 115,8 & 1,89 & 0,67 & 1,42 & 3,31 \\
\hline Herpothallon rubrocinctum & 48 & 83,6 & 1,36 & 0,80 & 1,71 & 3,07 \\
\hline Leptogium azureum & 57 & 63,2 & 1,03 & 0,95 & 2,03 & 3,06 \\
\hline Punctelia crispa & 26 & 122,1 & 1,99 & 0,43 & 0,92 & 2,91 \\
\hline Ricasolia discolor & 31 & 107,4 & 1,75 & 0,52 & 1,10 & 2,85 \\
\hline Heterodermia vulgaris & 51 & 53 & 0,86 & 0,85 & 1,81 & 2,68 \\
\hline Leptogium phyllocarpum & 38 & 79,2 & 1,29 & 0,63 & 1,35 & 2,64 \\
\hline Diorygma pruinosum & 46 & 46,6 & 0,76 & 0,77 & 1,64 & 2,40 \\
\hline Ramalina celastri & 42 & 34,6 & 0,56 & 0,70 & 1,49 & 2,06 \\
\hline Parmotrema consors & 30 & 51,6 & 0,84 & 0,50 & 1,07 & 1,91 \\
\hline Hemithecium chlorocarpum & 14 & 82 & 1,33 & 0,23 & 0,50 & 1,83 \\
\hline Brigantiaea leucoxantha & 36 & 31,7 & 0,52 & 0,60 & 1,28 & 1,80 \\
\hline Punctelia constantimontium & 23 & 58,1 & 0,95 & 0,38 & 0,82 & 1,76 \\
\hline Graphis obtectostriata & 22 & 55 & 0,90 & 0,37 & 0,78 & 1,68 \\
\hline Hypotrachyna livida & 28 & 36,9 & 0,60 & 0,47 & 1,00 & 1,60 \\
\hline Graphis calcea & 10 & 72,9 & 1,19 & 0,17 & 0,36 & 1,54 \\
\hline Parmelinopsis minarum & 20 & 45,6 & 0,74 & 0,33 & 0,71 & 1,45 \\
\hline Bacidia russeola & 20 & 44,2 & 0,72 & 0,33 & 0,71 & 1,43 \\
\hline Punctelia sp. 3 & 14 & 56,2 & 0,91 & 0,23 & 0,50 & 1,41 \\
\hline Heterodermia speciosa & 24 & 34,3 & 0,56 & 0,40 & 0,85 & 1,41 \\
\hline Heterodermia albicans & 24 & 33,7 & 0,55 & 0,40 & 0,85 & 1,40 \\
\hline Parmotrema clavuliferum & 19 & 41,7 & 0,68 & 0,32 & 0,68 & 1,35 \\
\hline Teloschistes exilis & 33 & 10,9 & 0,18 & 0,55 & 1,17 & 1,35 \\
\hline Parmotrema subcaperatum & 16 & 41,9 & 0,68 & 0,27 & 0,57 & 1,25 \\
\hline Heterodermia casarettiana & 16 & 41,7 & 0,68 & 0,27 & 0,57 & 1,25 \\
\hline
\end{tabular}


Tabela 2 (continuação)

\begin{tabular}{|c|c|c|c|c|c|c|}
\hline Espécies & NI & $\mathrm{CA}$ & $\mathrm{CR}$ & FA & FR & VI \\
\hline Leptogium involutum & 20 & 30,6 & 0,50 & 0,33 & 0,71 & 1,21 \\
\hline Dirinaria picta & 18 & 33,3 & 0,54 & 0,30 & 0,64 & 1,18 \\
\hline Crocodia clathrata & 23 & 18,9 & 0,31 & 0,38 & 0,82 & 1,13 \\
\hline Lecanora albella & 18 & 26,5 & 0,43 & 0,30 & 0,64 & 1,07 \\
\hline Bacidia fluminensis & 20 & 20,8 & 0,34 & 0,33 & 0,71 & 1,05 \\
\hline Parmotrema eciliatum & 10 & 41,6 & 0,68 & 0,17 & 0,36 & 1,03 \\
\hline Parmotrema subrugatum & 14 & 31,2 & 0,51 & 0,23 & 0,50 & 1,01 \\
\hline Parmotrema pilosum & 12 & 35,4 & 0,58 & 0,20 & 0,43 & 1,00 \\
\hline Graphis glauconigra & 12 & 33,8 & 0,55 & 0,20 & 0,43 & 0,98 \\
\hline Physcia aipolia & 20 & 12,9 & 0,21 & 0,33 & 0,71 & 0,92 \\
\hline Heterodermia leucomela & 16 & 18,2 & 0,30 & 0,27 & 0,57 & 0,87 \\
\hline Leptogium cyanescens & 14 & 21,2 & 0,35 & 0,23 & 0,50 & 0,84 \\
\hline Heterodermia lutescens & 12 & 24,1 & 0,39 & 0,20 & 0,43 & 0,82 \\
\hline Heterodermia diademata & 16 & 15,1 & 0,25 & 0,27 & 0,57 & 0,82 \\
\hline Parmotrema melanothrix & 9 & 28,2 & 0,46 & 0,15 & 0,32 & 0,78 \\
\hline Punctelia osorioi & 9 & 28,2 & 0,46 & 0,15 & 0,32 & 0,78 \\
\hline Phyllopsora breviuscula & 18 & 8,2 & 0,13 & 0,30 & 0,64 & 0,77 \\
\hline Usnea sp. 7 & 12 & 21,3 & 0,35 & 0,20 & 0,43 & 0,77 \\
\hline Lecanora achroa & 16 & 11,7 & 0,19 & 0,27 & 0,57 & 0,76 \\
\hline Usnea angulata & 11 & 21,1 & 0,34 & 0,18 & 0,39 & 0,73 \\
\hline Physcia tribacoides & 10 & 20,8 & 0,34 & 0,17 & 0,36 & 0,69 \\
\hline Parmotrema cf. eurysacum & 6 & 27 & 0,44 & 0,10 & 0,21 & 0,65 \\
\hline Usnea cf. rubicunda & 11 & 13,3 & 0,22 & 0,18 & 0,39 & 0,61 \\
\hline Pyxine subcinerea & 12 & 10,7 & 0,17 & 0,20 & 0,43 & 0,60 \\
\hline Malmidea hypomela & 11 & 12,7 & 0,21 & 0,18 & 0,39 & 0,60 \\
\hline Punctelia microsticta & 5 & 25,7 & 0,42 & 0,08 & 0,18 & 0,60 \\
\hline Ramalina sprengelii & 8 & 19,1 & 0,31 & 0,13 & 0,28 & 0,60 \\
\hline Malmidea fuscella & 11 & 9,9 & 0,16 & 0,18 & 0,39 & 0,55 \\
\hline Usnea sp. 2 & 10 & 11,8 & 0,19 & 0,17 & 0,36 & 0,55 \\
\hline Usnea sp. 1 & 11 & 9 & 0,15 & 0,18 & 0,39 & 0,54 \\
\hline Parmotrema recipiendum & 8 & 15,3 & 0,25 & 0,13 & 0,28 & 0,53 \\
\hline Graphis dolichographa & 9 & 12,4 & 0,20 & 0,15 & 0,32 & 0,52 \\
\hline Parmotrema rigidum & 10 & 9,9 & 0,16 & 0,17 & 0,36 & 0,52 \\
\hline Usnea sp. 9 & 12 & 5,2 & 0,08 & 0,20 & 0,43 & 0,51 \\
\hline Tephromela americana & 7 & 14 & 0,23 & 0,12 & 0,25 & 0,48 \\
\hline Leptogium isidiosellum & 7 & 12,4 & 0,20 & 0,12 & 0,25 & 0,45 \\
\hline Canoparmelia texana & 5 & 16,1 & 0,26 & 0,08 & 0,18 & 0,44 \\
\hline Parmotrema haitiense & 7 & 11,4 & 0,19 & 0,12 & 0,25 & 0,43 \\
\hline Graphis paraserpens & 3 & 19,6 & 0,32 & 0,05 & 0,11 & 0,43 \\
\hline
\end{tabular}


Tabela 2 (continuação)

\begin{tabular}{|c|c|c|c|c|c|c|}
\hline Espécies & NI & $\mathrm{CA}$ & $\mathrm{CR}$ & FA & FR & VI \\
\hline Lecanora concilianda & 6 & 12,9 & 0,21 & 0,10 & 0,21 & 0,42 \\
\hline Leptogium denticulatum & 5 & 13,9 & 0,23 & 0,08 & 0,18 & 0,40 \\
\hline Collema fasciculare & 9 & 5,1 & 0,08 & 0,15 & 0,32 & 0,40 \\
\hline Hypotrachyna polydactyla & 9 & 5,1 & 0,08 & 0,15 & 0,32 & 0,40 \\
\hline Pertusaria sp. 4 & 4 & 15 & 0,24 & 0,07 & 0,14 & 0,39 \\
\hline Cococcarpia erythroxyli & 9 & 3,8 & 0,06 & 0,15 & 0,32 & 0,38 \\
\hline Porina africana & 4 & 14,5 & 0,24 & 0,07 & 0,14 & 0,38 \\
\hline Usnea sp. 6 & 1 & 20,4 & 0,33 & 0,02 & 0,04 & 0,37 \\
\hline Normandina pulchella & 7 & 7,2 & 0,12 & 0,12 & 0,25 & 0,37 \\
\hline Ricasolia patinifera & 3 & 15,7 & 0,26 & 0,05 & 0,11 & 0,36 \\
\hline Punctelia hypoleucites & 6 & 9,1 & 0,15 & 0,10 & 0,21 & 0,36 \\
\hline Trypethelium nitidiusculum & 5 & 10,7 & 0,17 & 0,08 & 0,18 & 0,35 \\
\hline Physcia undulata & 7 & 6,2 & 0,10 & 0,12 & 0,25 & 0,35 \\
\hline Physcia sorediosa & 8 & 3,8 & 0,06 & 0,13 & 0,28 & 0,35 \\
\hline Lecanora grupo subfusca & 7 & 5,1 & 0,08 & 0,12 & 0,25 & 0,33 \\
\hline Grupo Lepraria sp. & 4 & 10,2 & 0,17 & 0,07 & 0,14 & 0,31 \\
\hline Pyxine daedalea & 6 & 5,8 & 0,09 & 0,10 & 0,21 & 0,31 \\
\hline Leptogium diaphanum & 6 & 5,7 & 0,09 & 0,10 & 0,21 & 0,31 \\
\hline Parmotrema catarinae & 4 & 9,9 & 0,16 & 0,07 & 0,14 & 0,30 \\
\hline Trypethelium quassiaecola & 4 & 9,8 & 0,16 & 0,07 & 0,14 & 0,30 \\
\hline Coenogonium byssothallinum & 7 & 2,3 & 0,04 & 0,12 & 0,25 & 0,29 \\
\hline Parmotrema praesorediosum & 5 & 6,4 & 0,10 & 0,08 & 0,18 & 0,28 \\
\hline Usnea sp. 3 & 6 & 3,9 & 0,06 & 0,10 & 0,21 & 0,28 \\
\hline Punctelia bolliana & 2 & 12,3 & 0,20 & 0,03 & 0,07 & 0,27 \\
\hline Haematomma africanum & 5 & 5,7 & 0,09 & 0,08 & 0,18 & 0,27 \\
\hline Graphis rimulosa & 2 & 12,2 & 0,20 & 0,03 & 0,07 & 0,27 \\
\hline Pertusaria carneola & 2 & 12 & 0,20 & 0,03 & 0,07 & 0,27 \\
\hline Platygramme caesiopruinosa & 5 & 4,8 & 0,08 & 0,08 & 0,18 & 0,26 \\
\hline Glyphis cicatricosa & 5 & 4,7 & 0,08 & 0,08 & 0,18 & 0,25 \\
\hline Parmotrema lindmanii & 3 & 8,6 & 0,14 & 0,05 & 0,11 & 0,25 \\
\hline Punctelia fimbriata & 4 & 6,3 & 0,10 & 0,07 & 0,14 & 0,24 \\
\hline Phlyctella brasiliensis & 5 & 4 & 0,07 & 0,08 & 0,18 & 0,24 \\
\hline Graphis subdisserpens & 5 & 3,9 & 0,06 & 0,08 & 0,18 & 0,24 \\
\hline Crocodia aff. clathrata & 4 & 6 & 0,10 & 0,07 & 0,14 & 0,24 \\
\hline Bacidia chapadensis & 5 & 3,8 & 0,06 & 0,08 & 0,18 & 0,24 \\
\hline Physcia poncinsii & 4 & 5,5 & 0,09 & 0,07 & 0,14 & 0,23 \\
\hline Parmotrema sancti-angeli & 3 & 7,2 & 0,12 & 0,05 & 0,11 & 0,22 \\
\hline Phyllopsora chlorophaea & 2 & 9,2 & 0,15 & 0,03 & 0,07 & 0,22 \\
\hline Parmotrema mellissii & 2 & 9 & 0,15 & 0,03 & 0,07 & 0,22 \\
\hline Phyllopsora buettneri & 5 & 2,4 & 0,04 & 0,08 & 0,18 & 0,22 \\
\hline Trichothelium angustiporum & 4 & 4,5 & 0,07 & 0,07 & 0,14 & 0,22 \\
\hline
\end{tabular}


Tabela 2 (continuação)

\begin{tabular}{|c|c|c|c|c|c|c|}
\hline Espécies & NI & $\mathrm{CA}$ & $\mathrm{CR}$ & FA & FR & VI \\
\hline Chapsa chionostoma & 4 & 4,4 & 0,07 & 0,07 & 0,14 & 0,21 \\
\hline Usnea sp. 8 & 1 & 10,8 & 0,18 & 0,02 & 0,04 & 0,21 \\
\hline Punctelia sp. 6 & 1 & 10,4 & 0,17 & 0,02 & 0,04 & 0,20 \\
\hline Coenogonium subdilutum & 3 & 5,8 & 0,09 & 0,05 & 0,11 & 0,20 \\
\hline Canoparmelia caroliniana & 4 & 3,4 & 0,06 & 0,07 & 0,14 & 0,20 \\
\hline Heterodermia pseudospeciosa & 4 & 3 & 0,05 & 0,07 & 0,14 & 0,19 \\
\hline Anthracothecium prasinum & 2 & 5,8 & 0,09 & 0,03 & 0,07 & 0,17 \\
\hline Ramalina usnea & 3 & 3,3 & 0,05 & 0,05 & 0,11 & 0,16 \\
\hline Bacidia subtestacea & 3 & 3,2 & 0,05 & 0,05 & 0,11 & 0,16 \\
\hline Bacidia alutacea & 2 & 5 & 0,08 & 0,03 & 0,07 & 0,15 \\
\hline Canoparmelia roseoreagens & 2 & 4,9 & 0,08 & 0,03 & 0,07 & 0,15 \\
\hline Chapsa sp. & 3 & 2,4 & 0,04 & 0,05 & 0,11 & 0,15 \\
\hline Usnea rubicunda & 2 & 4,3 & 0,07 & 0,03 & 0,07 & 0,14 \\
\hline Pyrenula pyrenuloides & 3 & 1,4 & 0,02 & 0,05 & 0,11 & 0,13 \\
\hline Pyxine cocoës & 3 & 1,3 & 0,02 & 0,05 & 0,11 & 0,13 \\
\hline Calopadia pruinosa & 2 & 3,3 & 0,05 & 0,03 & 0,07 & 0,12 \\
\hline Physcia sinuosa & 2 & 3,3 & 0,05 & 0,03 & 0,07 & 0,12 \\
\hline Pyrenula dissimulans & 1 & 5,4 & 0,09 & 0,02 & 0,04 & 0,12 \\
\hline Physcia krogiae & 3 & 1 & 0,02 & 0,05 & 0,11 & 0,12 \\
\hline Graphis puiggarii & 2 & 3,1 & 0,05 & 0,03 & 0,07 & 0,12 \\
\hline Graphis consanguinea & 2 & 2,6 & 0,04 & 0,03 & 0,07 & 0,11 \\
\hline Bapalmuia lafayettiana & 2 & 2,3 & 0,04 & 0,03 & 0,07 & 0,11 \\
\hline Phyllopsora confusa & 2 & 2,2 & 0,04 & 0,03 & 0,07 & 0,11 \\
\hline Punctelia sp. 4 & 2 & 1,7 & 0,03 & 0,03 & 0,07 & 0,10 \\
\hline Heterodermia flabellata & 2 & 1,6 & 0,03 & 0,03 & 0,07 & 0,10 \\
\hline Coenogonium pyrophthalmum & 2 & 1,5 & 0,02 & 0,03 & 0,07 & 0,10 \\
\hline Hyperphyscia adglutinata & 2 & 1,5 & 0,02 & 0,03 & 0,07 & 0,10 \\
\hline Psoroleglaena stigonemoides & 2 & 1,5 & 0,02 & 0,03 & 0,07 & 0,10 \\
\hline Heterodermia tremulans & 2 & 1,2 & 0,02 & 0,03 & 0,07 & 0,09 \\
\hline Opegrapha sp. 1 & 2 & 1,2 & 0,02 & 0,03 & 0,07 & 0,09 \\
\hline Parmelinopsis sp. & 2 & 1,2 & 0,02 & 0,03 & 0,07 & 0,09 \\
\hline Caloplaca erythranta & 2 & 1,1 & 0,02 & 0,03 & 0,07 & 0,09 \\
\hline Ochrolechia africana & 2 & 1,1 & 0,02 & 0,03 & 0,07 & 0,09 \\
\hline Phaeographis lecanographa & 2 & 1,1 & 0,02 & 0,03 & 0,07 & 0,09 \\
\hline Cococcarpia pellita & 2 & 1 & 0,02 & 0,03 & 0,07 & 0,09 \\
\hline Caloplaca sp. & 2 & 0,8 & 0,01 & 0,03 & 0,07 & 0,08 \\
\hline Hyperphyscia syncolla & 2 & 0,8 & 0,01 & 0,03 & 0,07 & 0,08 \\
\hline Punctelia graminicola & 1 & 2,9 & 0,05 & 0,02 & 0,04 & 0,08 \\
\hline Physcia erumpens & 2 & 0,6 & 0,01 & 0,03 & 0,07 & 0,08 \\
\hline Teloschistes cymbalifer & 2 & 0,6 & 0,01 & 0,03 & 0,07 & 0,08 \\
\hline Dirinaria confluens & 2 & 0,5 & 0,01 & 0,03 & 0,07 & 0,08 \\
\hline
\end{tabular}


Tabela 2 (continuação)

\begin{tabular}{|c|c|c|c|c|c|c|}
\hline Espécies & NI & $\mathrm{CA}$ & $\mathrm{CR}$ & FA & FR & VI \\
\hline Usnea sp. 5 & 2 & 0,4 & 0,01 & 0,03 & 0,07 & 0,08 \\
\hline Malmidea vinosa & 1 & 2,1 & 0,03 & 0,02 & 0,04 & 0,07 \\
\hline Parmotrema internexum & 1 & 2,1 & 0,03 & 0,02 & 0,04 & 0,07 \\
\hline Parmotrema tinctorum & 1 & 1,9 & 0,03 & 0,02 & 0,04 & 0,07 \\
\hline Ricasolia intermedia & 1 & 1,6 & 0,03 & 0,02 & 0,04 & 0,06 \\
\hline Punctelia riograndensis & 1 & 1,5 & 0,02 & 0,02 & 0,04 & 0,06 \\
\hline Parmotrema cf. rampoddense & 1 & 1,4 & 0,02 & 0,02 & 0,04 & 0,06 \\
\hline Pseudocyphellaria sp. & 1 & 1,4 & 0,02 & 0,02 & 0,04 & 0,06 \\
\hline Astrothelium crassum & 1 & 1,3 & 0,02 & 0,02 & 0,04 & 0,06 \\
\hline Pertusaria sp. 3 & 1 & 1,3 & 0,02 & 0,02 & 0,04 & 0,06 \\
\hline Leptogium cochleatum & 1 & 1,2 & 0,02 & 0,02 & 0,04 & 0,06 \\
\hline Usnea florida & 1 & 1,2 & 0,02 & 0,02 & 0,04 & 0,06 \\
\hline Anisomeridium albisedum & 1 & 1,1 & 0,02 & 0,02 & 0,04 & 0,05 \\
\hline Phyllopsora furfuracea & 1 & 1 & 0,02 & 0,02 & 0,04 & 0,05 \\
\hline Anisomeridium leptospermum & 1 & 0,9 & 0,01 & 0,02 & 0,04 & 0,05 \\
\hline Coenogonium nepalense & 1 & 0,9 & 0,01 & 0,02 & 0,04 & 0,05 \\
\hline Parmotrema muelleri & 1 & 0,9 & 0,01 & 0,02 & 0,04 & 0,05 \\
\hline Pertusaria sp. 1 & 1 & 0,9 & 0,01 & 0,02 & 0,04 & 0,05 \\
\hline Phyllopsora cf. santensis & 1 & 0,9 & 0,01 & 0,02 & 0,04 & 0,05 \\
\hline Cococcarpia stellata & 1 & 0,8 & 0,01 & 0,02 & 0,04 & 0,05 \\
\hline Lecanora thysanophora & 1 & 0,8 & 0,01 & 0,02 & 0,04 & 0,05 \\
\hline Pertusaria velata & 1 & 0,8 & 0,01 & 0,02 & 0,04 & 0,05 \\
\hline Pertusaria sp. 2 & 1 & 0,8 & 0,01 & 0,02 & 0,04 & 0,05 \\
\hline Physcia crispa & 1 & 0,8 & 0,01 & 0,02 & 0,04 & 0,05 \\
\hline Pyrenula тисоsа & 1 & 0,8 & 0,01 & 0,02 & 0,04 & 0,05 \\
\hline Graphis sp. 2 & 1 & 0,7 & 0,01 & 0,02 & 0,04 & 0,05 \\
\hline Parmotrema simulans & 1 & 0,7 & 0,01 & 0,02 & 0,04 & 0,05 \\
\hline Physcia alba & 1 & 0,7 & 0,01 & 0,02 & 0,04 & 0,05 \\
\hline Coenogonium interplexum & 1 & 0,6 & 0,01 & 0,02 & 0,04 & 0,05 \\
\hline Graphis tenella & 1 & 0,6 & 0,01 & 0,02 & 0,04 & 0,05 \\
\hline Leptogium austroamericanum & 1 & 0,6 & 0,01 & 0,02 & 0,04 & 0,05 \\
\hline Pertusaria flavens & 1 & 0,6 & 0,01 & 0,02 & 0,04 & 0,05 \\
\hline Hyperphyscia viridissima & 1 & 0,5 & 0,01 & 0,02 & 0,04 & 0,04 \\
\hline Carbacanthographis sp. & 1 & 0,4 & 0,01 & 0,02 & 0,04 & 0,04 \\
\hline Heterodermia africana & 1 & 0,3 & 0,00 & 0,02 & 0,04 & 0,04 \\
\hline Heterodermia magellanica & 1 & 0,3 & 0,00 & 0,02 & 0,04 & 0,04 \\
\hline Rinodina conradii & 1 & 0,3 & 0,00 & 0,02 & 0,04 & 0,04 \\
\hline Usnea sp. 4 & 1 & 0,3 & 0,00 & 0,02 & 0,04 & 0,04 \\
\hline Parmotrema austrosinense & 1 & 0,2 & 0,00 & 0,02 & 0,04 & 0,04 \\
\hline Punctelia subpraesignis & 1 & 0,2 & 0,00 & 0,02 & 0,04 & 0,04 \\
\hline \multirow[t]{2}{*}{ Teloschistes flavicans } & 1 & 0,2 & 0,00 & 0,02 & 0,04 & 0,04 \\
\hline & & 6142,9 & 100,0 & 46,85 & 100,0 & 200,0 \\
\hline
\end{tabular}


Do grupo morfológico folioso, composto pelas espécies H. obscurata, Punctelia sp. 5 e R. erosa citadas anteriormente apresentaram os maiores valores de importância, cobertura absoluta e número de indivíduos. Da família Parmeliaceae, se destaca ainda a espécie Parmotrema reticulatum que ocupou o sexto maior valor de importância na comunidade $(\mathrm{VI}=7,89)$ e no número de indivíduos $(\mathrm{NI}=102)$.

O grupo morfológico fruticoso também se destacou na comunidade, com $13,88 \%$ do VI total $\left(\sum=200\right)$. As espécies Ramalina peruviana e $R$. celastri pertencentes a este grupo, apresentaram os maiores valores de importância $(\mathrm{VI}=4,71 \mathrm{eVI}=2,06)$ e número de indivíduos, 68 e 42 respectivamente. Ressalta-se também o gênero Usnea que obteve a maior representatividade $(4,88 \%)$.

Os liquens que portam cianobactérias tiveram baixo percentual no valor de importância na comunidade, apenas $9,93 \%$ do VI total $\left(\sum=200\right)$. As espécies deste grupo que se destacaram na comunidade pelo número de indivíduos foram Leptogium azureum $(\mathrm{NI}=57)$ e L. phyllocarpum $(\mathrm{NI}=38)$.

\section{Discussão}

A elevada riqueza de espécies para as áreas florestais ribeirinhas da região sul da APA do Ibirapuitã associada aos novos registros de espécies para a ciência, Brasil e o Estado do Rio Grande do Sul demonstra a carência de estudos, principalmente de cunho ecológico, para a região.

As famílias Parmeliace, Physciaceae e Graphidaceae se destacaram pela maior representatividade de espécies na comunidade liquênica da APA do Ibirapuitã. As duas primeiras são predominantes nas paisagens liquênicas, onde espécimes de Parmeliaceae são característicos de bordas de mata com maior luminosidade e vento (Marcelli 1998). Esta é a família liquênica que detém a maior dominância e riqueza de espécies para o país. De acordo com Blanco et al. (2005) estima-se que existam, aproximadamente, 2.400 espécies distribuídas em 85 gêneros. Nossos estudos corroboram com outros trabalhos realizados em áreas florestais do Estado (Käffer \& Martins-Mazzitelli 2005, Martins et al. 2008, Käffer et al. 2009, 2010, Martins \& Marcelli 2011, Martins et al. 2011, Koch et al. 2012). Graphidaceae é a segunda maior família de fungos liquenizados e dominam grande parte da comunidade liquênica das regiões tropicais, com mais de 1.800 espécies conhecidas (Rivas-Plata et al.
2013). Kaffer et al. (2011) em trabalho realizado em área urbana na região sul do Brasil, citou esta família como a terceira em número de espécies. Para a APA do Ibirapuitã, Käffer et al. (2014 b, 2015) registraram nove espécies de Graphis como novas ocorrências para o Brasil e Estado do Rio Grande do Sul, além de uma nova espécie, sendo que esta obteve o $24^{\circ}$ maior valor de importância na comunidade (VI = 1,68).

Nas áreas florestais ribeirinhas foram registrados baixo percentual de liquens que portam cianobactérias, estes normalmente estão relacionados à ambientes úmidos e sombreados. Espécies do gênero Leptogium foram mais frequentes em forófitos localizados no interior das áreas florestais ribeirinhas. Espécies deste gênero representaram $4,50 \%$ do valor de importância da comunidade, com destaque para Leptogium azureum que obteve o maior número de indivíduos da família $(\mathrm{NI}=57)$. As florestas ribeirinhas analisadas da região sul da APA do Ibirapuitã se caracterizam pela vegetação de médio porte, sub-bosque variando de denso a esparso e pela presença de curso d' água. Segundo Renhorn et al. (1997), as espécies que portam cianobactérias como fotobionte são geralmente sensíveis ao estresse ambiental como a borda de uma floresta. O gênero Leptogium é um dos maiores dentro da família Collemataceae, que compreende os foliosos grandes, crescem nas partes úmidas de vários ambientes, desde o nível do mar até 3.400 metros de altitude, sendo que o grau de especificidade apresentada em relação ao substrato é bastante variável de acordo com a espécie. Possuem ampla distribuição, mas algumas espécies são restritas em regiões tropicais onde a diversidade do gênero é grande (Sierk 1964, Swinscow \& Krog 1988, Otalora et al. 2014).

As espécies com maior valor de importância na comunidade estão relacionadas às suas características ecológicas. Heterodermia obscurata apresentou o maior valor de importância obtendo também elevada frequência absoluta $(6,5)$, cobertura relativa $(13,87 \%)$ e maior número de indivíduos (390), apesar de seu talo pequeno. Esta espécie pertence à família Physciaceae que, de acordo com Marcelli (1998), é a segunda maior família em número de espécies no Brasil. As espécies do gênero Heterodermia ocorrem em locais relativamente abertos, com boa luminosidade, como beira de mata, mata aberta e clareiras (Martins 2007). A região da Campanha apresenta um número considerável de espécies do gênero Heterodermia, compreendendo metade das ocorrentes no Estado do Rio Grande do Sul (Eliasaro 1992). 
As espécies Punctelia sp. 5 e Parmotrema cetratum ocuparam o segundo e terceiro maior valor de importância na comunidade por apresentarem maiores valores de cobertura e frequência absoluta, respectivamente e, ambas pertencem à família Parmeliaceae. Espécies do gênero Parmotrema se caracterizam por apresentar talos geralmente grandes (Spielmann \& Marcelli 2008), enquanto que espécimes de Punctelia podem variar de 3,0 a $20 \mathrm{~cm}$ de diâmetro (Canêz 2009). Este último gênero tem seu centro de dispersão na África e América do Sul (Elix 1994) e o RS é o Estado com maior diversidade de espécies (Canêz 2009). Em todas as áreas analisadas foram registradas espécies de Parmotrema e Punctelia cobrindo grandes extensões de troncos, galhos e/ou ramos.

Outra espécie de destaque na comunidade foi Ricasolia erosa que ocupou o terceiro maior valor de importância. Liquens foliosos de talo grande como $R$. erosa são mais comuns em áreas preservadas ou mais antigas do que em florestas mais jovens. Muitos trabalhos têm demonstrado que isto ocorre devido ao lento crescimento dos liquens, ao fato do microhabitat dessas florestas serem mais estáveis, e pela baixa eficiência de dispersão das espécies (Hinds \& Hinds 2007). Espécies deste gênero têm sido citadas como indicadoras de ecossistemas florestais preservados e associadas a áreas de floresta de elevada continuidade ecológica (Campbell \& Fredeen 2004, Liira \& Sepp 2009). Sua ocorrência também está associada com uma variedade de outras espécies raras ou em extinção, podendo ser considerada como uma espécie de guarda-chuva (Nascimbene et al. 2010). Espécies do gênero Ricasolia foram registradas em $80 \%$ das áreas florestais ribeirinhas analisadas e presentes em $61,7 \%$ dos forófitos amostrados.

O grupo morfológico fruticoso foi também de grande relevância na estrutura da comunidade, contribuindo com 21 táxons e perfazendo um total de 245 indivíduos, com destaque para os gêneros Usnea e Ramalina. Espécies deste grupo foram mais frequentes nos níveis mais elevados do tronco (acima de $100 \mathrm{~cm}$ de altura do solo), em forófitos localizados próximos a bordas das matas, em áreas florestais mais abertas, onde o sub-bosque se encontrava mais esparso e/ou em árvores isoladas. Também foram registrados muitos espécimes em galhos e ramos das árvores no levantamento florístico. Os liquens fruticosos são mais abundantes em ambientes mais abertos e ensolarados (Wodal 2006, Fleig \& Grüninger 2008) e são considerados mais sensíveis a alterações ambientais (Hawksworth et al. 2005). Espécies do gênero Usnea possuem ampla distribuição em regiões tropicais e temperadas do mundo (Knight 2014). E em regiões mais úmidas dos trópicos, este gênero produz uma abundância de morfo e quimiotipos (Swinscow \& Krog 1979).

O grande número de espécies, especialmente os novos registros, vem a contribuir com os estudos da liquenologia nas florestas ribeirinhas na APA do Ibirapuitã e para a região da campanha, no sul do Brasil. A boa qualidade ambiental de algumas áreas florestais, especialmente aquelas localizadas na Estância São Maurício e Fazenda Rincão Bonito (Käffer et al. 2014 a), a disponibilidade de forófitos adequados e as condições microclimáticas das áreas florestais são fatores que contribuíram para os resultados encontrados.

Os resultados deste estudo poderão ser utilizados como subsídio para a elaboração do Plano de manejo da área da APA do Ibirapuitã e contribuir para o conhecimento da liquenobiota do Estado do Rio Grande do Sul e do Brasil. E, a preservação das florestas ribeirinhas nas áreas analisadas é de vital importância para assegurar não somente a biodiversidade, representada pela variabilidade genética existente nas populações naturais, como também para a proteção dos cursos d' água do rio Ibirapuitã e seus afluentes.

\section{Agradecimentos}

Agradecemos aos liquenólogos Dr. André Aptroot da ABL Herbarium, Holanda, Dra. Marcela E. da S. Cáceres da Universidade Federal de Sergipe, Dr. Marcos J. Kitaura do Instituto de Biociências da UNESP e Dra. Luciana Canêz da Universidade Federal do Mato Grosso do Sul, pela identificação e/ou confirmação de algumas espécies crostosas e foliosas; ao Museu de Ciências Naturais da Fundação Zoobotânica do Rio Grande do Sul, por oferecer as dependências para as atividades de laboratório; e ao Conselho Nacional de Desenvolvimento Científico e Tecnológico - CNPq (processo número 160115/2012-4), pela bolsa de Pós-Doc do primeiro Autor.

\section{Literatura citada}

Andrade, R.O. 2013. Intervenções sustentáveis. Revista da FAPESP on line, 206, São Paulo, pp. 52. Disponível em http://www.revistapesquisa.fapesp.br/ BiotaFapesp. pdf. (acesso em 19-VIII-2013). 
Backes, A. 2012. Áreas protegidas no estado do Rio Grande do Sul: o esforço para a conservação. Pesquisas Botânica 63: 225-355.

Blanco, O., Crespo A., Divakar, P.K., Elix, J.A. \& Lumbsch, H.T. 2005. Molecular phylogeny of parmotremoid lichens (Ascomycota, Parmeliaceae). Mycologia, 97: 150-159.

Boldrini, I. 2009. A flora dos Campos do Rio Grande do Sul. In: V.D. Pillar, S.C. Müller, Z.M.S. Castilhos, A.V.A. Jacques (eds.). Campos Sulinos: Conservação e Uso Sustentável da Biodiversidade. Ministério do Meio Ambiente. Brasília, pp. 63-77.

Brunialti, G. \& Giordani, P. 2003.Variability of lichen diversity in a climatically heterogeneous area (Liguria, NW Italy). Lichenologist 35: 55-69.

Cáceres, M.E., Lücking, R. \& Rambold, G. 2008. Corticolous microlichens in northeastern Brazil: habitat differentiation between coastal Mata Atlântica, Caatinga and Brejos de Altitude. The Bryologist 111: 98-117.

Campbell, J. \& Fredeen A.L. 2004. Lobaria pulmonaria abundance as an indicator of macrolichen diversity in Interior Cedar-Hemlock forests of east-central British Columbia. Canadian Journal of Botany 82: 970-982.

Canêz, L.S. 2009. Estudos taxonômicos em Punctelia (Parmeliaceae, Ascomycetes Liquenizados). Tese de Doutorado, Instituto de Botânica, São Paulo.

Eliasaro, S. 1992. Liquens do gênero Heterodermia (Pyxinaceae-Ascomycotina) no Rio Grande do Sul, Brasil. Dissertação de Mestrado, Universidade Federal do Rio Grande do Sul, Porto Alegre.

Elix, J.A. 1994. Parmeliaceae. Flora of Australia 55: 1-360.

Esseen, P.A., Renhorn, K.E. \& Pettersson, R.B. 1996. Epiphytic lichen biomass in managed and old-growth boreal forests: effect of branch quality. Ecology Applied 6: 228-238.

Filgueiras, T.S., Nogueira, P.E., Brochado, A.L. \& Guala II, G. F. 1994. Caminhamento - Um método expedito para levantamentos florísticos qualitativos. Cadernos de Geociências 12: 39-43.

Fleig, M. \& Grüninger, W. 2008. Liquens da Floresta com Araucária no Rio Grande do Sul. Pró-Mata: Guia de Campo 3. University of Tübingen, Germany.

Giordani, P., Brunialti, G., Bacaro, G. \& Nascimbene, J. 2012. Functional traits of epiphytic lichens as potential indicators of environmental conditions in forest ecosystems. Ecological Indicators 18:413-420.

Gunnarsson, B., Hake, M. \& Hultengren, S. 2004. A functional relationship between species richness of spiders and lichen in spruce. Biodiversity and Conservation 13: 685-693.

Hawksworth, D.L., Iturriaga, T. \& Crespo, A. 2005. Líquenes como bioindicadores inmediatos de contaminación y cambios medio-ambientales en los trópicos. Revista Iberoamericano Micology 22: 71-82.
Hinds, J.W. \& Hinds, P.L. 2007. The macrolichens of New England. Memoirs of the New York Botanical Garden 96. The New York Botanical Garden Press. New York, USA.

Jüriado, I., Paal, J. \& Liira, J. 2003. Epiphytic and epixylic lichen species diversity in Estonian natural forests. Biodiversity and Conservation 12: 1587-1607.

Liira, J. \& Sepp, T. 2009. Indicators of structural and habitat natural quality in boreo-nemoral forests along the management gradient. Annals Botanny Fennici 46: 308-325

Käffer, M.I. \& Martins-Mazzitelli, S.M.A. 2005. Fungos liquenizados corticícolas e terrícolas da área da sub-bacia do Sinos e Taquari-Antas, RS, Brasil. Acta Botanica Brasilica, 19: 813-817.

Käffer, M.I., Ganade, G. \& Marcelli, M.P. 2009. Lichen diversity and composition in Araucaria forests and tree monocultures in southern Brazil. Biodiversity and Conservation 18: 3543-3561.

Käffer, M.I., Marcelli, M.P. \& Ganade, G. 2010. Distribution and composition of the lichenized mycota on a landscape mosaic from southern Brazil. Acta Botanica Brasilica 24: 790-802.

Käffer, M.I., Alves, C., Caceres, M.E., Martins, S.M.A. \& Vargas, V.M.F. 2011. Caracterização da comunidade liquênica corticícola de Porto Alegre e áreas adjacentes, RS, Brasil. Acta Botanica Brasilica, 25: 832-844.

Käffer, M.I. \& Martins, S.M.A. 2014a. Evaluation of the environmental quality of a protected riparian forest in Southern Brazil. Bosque (Valdivia. Impresa) 35: 325-331.

Käffer, M.I., Martins, S.M.A., Caceres, M.E.S. \& Aptroot, A. 2014b. A new, locally common Graphis (Graphidaceae) species from southern Brazil. Cryptogamie. Mycologie, 35: 233 - 237.

Käffer, M.I., Koch, N.M., Aptroot, A. \& Martins, S.M.A. 2015. New records of corticolous lichens for South America and Brazil. Plant Ecology and Evolution 148: 111-118.

Knight, A. 2014. Lichens of New Zealand. An Introductory illustrated guide. Dunebin: New Zealand. pp. 1-56.

Koch, N.M., Maluf, R.W. \& Martins, S.M.A. 2012. Comunidade de liquens foliosos em Piptocarpha angustifolia Dusén ex Malme (Asteraceae) em área de Floresta Ombrófila Mista no estado do Rio Grande do Sul, Brasil. Iheringia, Sér. Bot. 67: 47-57.

Marcelli, M.P. 1998. History and current knowledge of Brazilian lichenology. In: M.P. Marcelli \& M.R.D. Seaward (ed.). Lichenology in Latin America: history, current knowledge and application. São Paulo, CETESB: SMA, pp. 25-45.

Martins, M.F.N. 2007. O gênero Heterodermia (Physciaceae, Ascomycota liquenizados) no Estado de São Paulo, Brasil. Universidade Estadual de São Paulo, Botucatu, SP. 
Martins, S.M.A. \& Marcelli, M.P. 2011. Specific distribution of lichens on Dodonaea viscosa L. in the restinga area of Itapuã State Park in Southern Brazil. Hoehnea 38: 397-411.

Martins, S.M.A., Käffer, M.I. \& Lemos, A. 2008. Liquens como bioindicadores da qualidade do ar numa área de termoelétrica, Rio Grande do Sul, Brasil. Hoehnea $35: 425-433$.

Martins, S.M.A., Käffer, M.I., Alves, C.R. \& Pereira, V.C. 2011. Fungos liquenizados da Mata Atlântica, no sul do Brasil. Acta Botanica Brasilica 25: 286-292.

McCune, B. 1993. Gradients in epiphyte biomass in three Pseudotsuga-Tsuga forests of different ages in western Oregon and Washington. Bryologist 96: 405-411.

Nascimbene, J., Benesperi, R., Brunialti, G., Catalano, I., Vedove, M.D., Grillo, M., Isocrono, D., Matteucci, E., Potenza, G., Puntillo, D., Puntillo, M., Ravera, S., Rizzi, G. \& Giordani, P. 2013. Patterns and drivers of b-diversity and similarity of Lobaria pulmonaria communities in Italian forests. Journal Ecology 101: 493-505.

Otálora, M.A.G., Jørgensen, P.M. \& Wedin, M. 2014. A revised generic classification of the jelly lichens, Collemataceae. Fungal Diversity, 64: 275-293.

Renhorn, K.E., Esseen, P.A., Palmqvist, K. \& Sundberg, B. 1997. Growth and vitality of epiphytic lichens: I. Responses to microclimate along a forest edge-interior gradient. Oecologia, 109: 1-9.

Richardson D.H.S. \& Cameron, R.P. 2004. Cyanolichens: their response to pollution and possible management strategies for their conservation in northeastern North America. Northeast Naturalist 11: 1-22.
Rivas-Plata, E., Parnmen, S., Staiger, B., Mangold, A., Frisch, A., Weerakoon, G., Hernández, J.E. Cáceres, M., M.E.S., Kalb, K., Sipman, H.J.M., Common, R.S., Nelsen, M.P., Lücking, R. \& Lumbsch. H.T. 2013. A molecular phylogeny of Graphidaceae (Ascomycota: Lecanoromycetes: Ostropales) including 428 species. MycoKeys 6: 55-64.

Sierk, H.A. 1964. The genus Leptogium in north America and north of Mexico. Bryologist 67: 1-317.

Spielmann, A.A. \& Marcelli, M.P. 2008. Parmeliaceae (Ascomycota liquenizados) nos barrancos e peraus da encosta da Serra Geral, Vale do rio Pardo, Rio Grande do Sul, Brasil. I. Introdução e chave para gêneros. Iheringia, Série Botânica 63: 159-169.

Swinscow, T.D.V. \& Krog, H. 1979. The fruticose species of Usnea subgenus Usnea in east Africa. Lichenologis 11: $207-252$.

Swinscow, T.D.V. \& Krog, H. 1998. Macrolichens of East Africa. British Museum (Natural History) London.

Tuvi, E.L., Vellak, A., Reier, Ü., Szava-Kovats, R. \& Pärtel, M. 2011. Establishment of protected areas in different ecoregions, ecosystems, and diversity hotspots under successive political systems. Biological Conservation 144: 1726-1732.

Valencia M.C. de, Ceballos J.A. 2002. Hongos liquenizados. Universidad Nacional de Colombia, Bogotá.

Werth S. 2001. Key factors for epiphytic macrolichen vegetation in deciduous forests of Troms county, northern Norway: human impact, substrate, climate or spatial variation? Thesis, Department of Biology, Faculty of Science, University of Tromsø, Tromsø, Norway.

Wodal, C., Huber A. \& Dohrenbuschl, A. 2006. Vegetación epífita y captación de neblina en bosques siempre verdes en la Cordillera Pelada, Sur de Chile. Bosque 24: 231-240.

World Bird Watch. 2012. Protecting the Pampas. June 2012. Disponível em http://www.savebrasil.org,br (acesso em 22-VIII-2013). 